\title{
A BOUNDARY VALUE PROBLEM FOR SECOND-ORDER NONLINEAR DIFFERENCE EQUATIONS ON THE INTEGERS
}

\author{
F. DAL ${ }^{1}$ and G. SH. GUSEINOV ${ }^{2}$
}

(Received 27 June, 2003; revised 26 July, 2005)

\begin{abstract}
In this study, we are concerned with a boundary value problem (BVP) for nonlinear difference equations on the set of all integers $\mathbb{Z}$, under the assumption that the left-hand side is a second-order linear difference expression which belongs to the so-called Weyl-Hamburger limit-circle case. The BVP is considered in the Hilbert space $\ell^{2}$ and includes boundary conditions at infinity. Existence and uniqueness results for solution of the considered BVP are established.
\end{abstract}

\section{Introduction}

Let $\Delta$ be the forward difference operator defined by $\Delta y(t)=y(t+1)-y(t)$. Consider the second-order nonlinear difference equation

$$
\Delta[p(t-1) \Delta y(t-1)]+q(t) y(t)=f(t, y(t)), \quad t \in \mathbb{Z},
$$

where $p(t)$ and $q(t)$ are real-valued functions defined on $\mathbb{Z}, p(t) \neq 0$ for all $t \in \mathbb{Z}$ and $f(t, \xi)$ is a real-valued function defined on $\mathbb{Z} \times \mathbb{R}$. Note that by $\mathbb{Z}$ we denote the set of integers and by $\mathbb{R}$ the set of real numbers.

Denote by $\ell^{2}$ the Hilbert space of real-valued functions $y(t)$ on $\mathbb{Z}$ such that $\sum_{t=-\infty}^{\infty}|y(t)|^{2}<\infty$ with the inner product $(y, z)=\sum_{t=-\infty}^{\infty} y(t) z(t)$. Further, we let

$$
L y(t):=\Delta[p(t-1) \Delta y(t-1)]+q(t) y(t)
$$

and denote by $D$ the linear manifold of all elements $y \in \ell^{2}$ such that $L y \in \ell^{2}$. Notice that all functions $y(t)$ with finite support (that is, functions having only finitely many nonzero values) belong to $D$.

\footnotetext{
'Department of Mathematics, Ege University, 35100 Bornova, Izmir, Turkey.

${ }^{2}$ Department of Mathematics, Atilim University, 06836 Incek, Ankara, Turkey; e-mail:

guseinov@atilim.edu.tr.

(C) Australian Mathematical Society 2005, Serial-fee code 1446-1811/05
} 
Let us set $y^{(\Delta)}(t)=p(t) \Delta y(t)$ and for two arbitrary functions $y=y(t)$ and $z=z(t)$ we define the Wronskian of $y$ and $z$ by

$$
W_{t}(y, z)=y(t) z^{[\Delta]}(t)-y^{[\Delta]}(t) z(t)=p(t)[y(t) z(t+1)-y(t+1) z(t)], \quad t \in \mathbb{Z} .
$$

It follows from Green's formula

$$
\sum_{i=a}^{b}[(L y) z-y(L z)](t)=W_{a-1}(y, z)-W_{b}(y, z) \quad(a, b \in \mathbb{Z}, \quad a \leq b)
$$

that, for all $y, z \in D$, the limits $W_{-\infty}(y, z)=\lim _{t \rightarrow-\infty} W_{t}(y, z)$ and $W_{\infty}(y, z)=$ $\lim _{t \rightarrow \infty} W_{t}(y, z)$ exist as finite numbers.

We will assume that the following conditions are satisfied.

(C1) The coefficients $p(t)$ and $q(t)$ are such that all solutions of the second-order linear difference equation

$$
\Delta[p(t-1) \Delta y(t-1)]+q(t) y(t)=0, \quad t \in \mathbb{Z},
$$

belong to $\ell^{2}$.

(C2) For each $t \in \mathbb{Z}$ the function $f(t, \xi)$ is continuous in $\xi \in \mathbb{R}$, and

$$
|f(t, \xi)| \leq g(t)+d|\xi|
$$

for all $(t, \xi)$ in $\mathbb{Z} \times \mathbb{R}$, where $g(t) \geq 0, g \in \ell^{2}$ and $d$ is a positive constant.

REMARK. The condition ( $\mathrm{C} 1$ ) means that for the difference expression (1.2) the socalled Weyl-Hamburger limit-circle case holds (see [5, Chapter 7]). If, for example, $p(t)=t^{2}+1$ and $q(t)=2 t^{2}-2 t+3$, then the condition (C1) holds.

REMARK. If we define the operator $F$ taking each function $y(t)$ to the function $f(t, y(t))$, then the condition (1.5) is necessary and sufficient for $F$ to map $\ell^{2}$ into itself (see [6]).

Let $u=u(t)$ and $v=v(t)$ be solutions of (1.4) satisfying the initial conditions

$$
u(0)=0, \quad u^{[\Delta]}(0)=1 ; \quad v(0)=-1 \quad \text { and } \quad v^{[\Delta]}(0)=0 .
$$

By the constancy of the Wronskian of any two solutions of (1.4) we have $W_{t}(u, v)=1$. Consequently, $u$ and $v$ are linearly independent and they form a fundamental system of solutions of (1.4). It follows from the condition (C1) that $u, v \in \ell^{2}$ and moreover $u, v \in D$. Therefore for each $y \in D$ the values $W_{ \pm \infty}(y, u)$ and $W_{ \pm \infty}(y, v)$ exist and 
are finite. For these values we can get, by using Green's formula (1.3) and the initial conditions (1.6), the formulas

$$
\begin{aligned}
& W_{-\infty}(y, u)=y(0)+\sum_{-\infty}^{t=0} u(t) L y(t), \\
& W_{-\infty}(y, v)=y^{[\Delta]}(0)+\sum_{-\infty}^{t=0} v(t) L y(t), \\
& W_{\infty}(y, u)=y(0)-\sum_{t=1}^{\infty} u(t) L y(t), \\
& W_{\infty}(y, v)=y^{[\Delta]}(0)-\sum_{t=1}^{\infty} v(t) L y(t) .
\end{aligned}
$$

Our boundary value problem (BVP) consists of finding a function $y=y(t), t \in \mathbb{Z}$, such that $y \in \ell^{2}$ and $y$ satisfies (1.1) [consequently $y \in D$ by (1.5)] and the boundary conditions at $-\infty$ and $\infty$ :

$$
\alpha W_{-\infty}(y, u)+\beta W_{-\infty}(y, v)=d_{1} \quad \text { and } \quad \gamma W_{\infty}(y, u)+\delta W_{\infty}(y, v)=d_{2},
$$

where $\alpha, \beta, \gamma$, and $\delta$ are given real numbers satisfying the condition (C3) $\omega:=\alpha \delta-\beta \gamma \neq 0$, and $d_{1}, d_{2}$ are arbitrary given real numbers.

Notice that since in (1.8) the function $y$ satisfies (1.1), from (1.7) we have the following formulas for the values $W_{ \pm \infty}(y, u)$ and $W_{ \pm \infty}(y, v)$ :

$$
\begin{aligned}
& W_{-\infty}(y, u)=y(0)+\sum_{-\infty}^{t=0} u(t) f(t, y(t)), \\
& W_{-\infty}(y, v)=y^{[\Delta]}(0)+\sum_{-\infty}^{t=0} v(t) f(t, y(t)), \\
& W_{\infty}(y, u)=y(0)-\sum_{t=1}^{\infty} u(t) f(t, y(t)), \\
& W_{\infty}(y, v)=y^{[\Delta]}(0)-\sum_{t=1}^{\infty} v(t) f(t, y(t)) .
\end{aligned}
$$

In Section 2 we construct an appropriate Green's function by means of which the BVP (1.1), (1.8) is reduced to a fixed point problem.

In Section 3 by using the Contraction Mapping theorem (Banach Fixed Point theorem) we show that there is a unique solution of the BVP $(1.1),(1.8)$ if $f(t, \xi)$ satisfies a Lipschitz condition. 
In Section 4 a theorem based on the Schauder Fixed Point theorem is proved which gives a result that yields existence of solutions without the implication that solutions must be unique.

Finally note that a similar problem in the case of the discrete semi-axis was earlier investigated in [7] and in the continuous case in [8]. For other formulations of BVPs on infinite intervals we refer to [1-4].

\section{Green's function and the operator $A$}

For $h \in \ell^{2}$ consider the linear BVP

$$
\begin{aligned}
\Delta[p(t-1) \Delta y(t-1)]+q(t) y(t) & =h(t), \quad t \in \mathbb{Z}, \\
\alpha W_{-\infty}(y, u)+\beta W_{-\infty}(y, v) & =0, \\
\gamma W_{\infty}(y, u)+\delta W_{\infty}(y, v) & =0
\end{aligned}
$$

where $y \in \ell^{2}$ is a desired solution and $u$ and $v$ are solutions of (1.4) under the initial conditions (1.6).

Let us set

$$
\varphi(t)=\alpha u(t)+\beta v(t) \text { and } \psi(t)=\gamma u(t)+\delta v(t) .
$$

These functions (together with $u$ and $v$ ) are solutions of (1.4) and are in $\ell^{2}$. In addition we have, for all $t \in \mathbb{Z}$,

$$
\begin{array}{ll}
W_{t}(\varphi, u)=\varphi(0)=-\beta, & W_{t}(\varphi, v)=\varphi^{[\Delta]}(0)=\alpha \\
W_{t}(\psi, u)=\psi(0)=-\delta, & W_{t}(\psi, v)=\psi^{[\Delta]}(0)=\gamma
\end{array}
$$

Therefore $\varphi$ satisfies the boundary condition at $-\infty$ in (2.2), and $\psi$ satisfies the boundary condition at $\infty$. The Wronskian of $\varphi$ and $\psi$ is

$$
W_{t}(\varphi, \psi)=\alpha \delta-\beta \gamma=\omega
$$

and hence it is different from zero by the condition (C3). Let

$$
G(t, s)=\frac{1}{\omega} \begin{cases}\varphi(t) \psi(s) & \text { if }-\infty<t \leq s<\infty \\ \varphi(s) \psi(t) & \text { if }-\infty<s \leq t<\infty\end{cases}
$$

Since $\varphi, \psi \in \ell^{2}$, we have

$$
\sum_{t=-\infty}^{\infty} \sum_{s=-\infty}^{\infty}|G(t, s)|^{2}<\infty
$$


THEOREM 2.1. The nonhomogeneous BVP (2.1)-(2.2) has a unique solution $y \in \ell^{2}$ for which the formula

$$
y(t)=\sum_{s=-\infty}^{\infty} G(t, s) h(s), \quad t \in \mathbb{Z}
$$

holds, where the function $G(t, s)$ is defined by $(2.7)$, and $G(t, s)$ is called the Green's function of the BVP (2.1)-(2.2).

PROOF. Under the condition (C3), by (2.6), the solutions $\varphi(t)$ and $\psi(t)$ of the homogeneous equation (1.4) are linearly independent. Consider the function

$$
z(t)=\frac{1}{\omega} \sum_{-\infty}^{s=t-1}[\varphi(s) \psi(t)-\varphi(t) \psi(s)] h(s), \quad t \in \mathbb{Z} .
$$

Since $\varphi, \psi$, and $h$ belong to $\ell^{2}$, the series (2.10) is convergent for each $t \in \mathbb{Z}$. It can be directly verified that the function $z(t)$ defined by $(2.10)$ is a particular solution of the nonhomogeneous equation (2.1). Therefore the general solution of (2.1) has the form

$$
y(t)=c_{1} \varphi(t)+c_{2} \psi(t)+\frac{1}{\omega} \sum_{-\infty}^{s=t-1}[\varphi(s) \psi(t)-\varphi(t) \psi(s)] h(s)
$$

where $c_{1}$ and $c_{2}$ are arbitrary constants. Now we try to choose the constants $c_{1}$ and $c_{2}$ so that the function $y(t)$ also satisfies the boundary conditions (2.2). From (2.11) we have

$$
y^{[\Delta]}(t)=c_{1} \varphi^{[\Delta]}(t)+c_{2} \psi^{[\Delta]}(t)+\frac{1}{\omega} \sum_{-\infty}^{s=t}\left[\varphi(s) \psi^{[\Delta]}(t)-\varphi^{[\Delta]}(t) \psi(s)\right] h(s) .
$$

Therefore, taking (2.4) and (2.5) into account, we find

$$
\begin{aligned}
W_{t}(y, u) & =y(t) u^{[\Delta]}(t)-y^{[\Delta]}(t) u(t) \\
& =c_{1} W_{t}(\varphi, u)+c_{2} W_{t}(\psi, u)+\frac{1}{\omega} \sum_{-\infty}^{s=t}\left[\varphi(s) W_{t}(\psi, u)-\psi(s) W_{t}(\varphi, u)\right] h(s) \\
& =-c_{1} \beta-c_{2} \delta+\frac{1}{\omega} \sum_{-\infty}^{s=t}[-\delta \varphi(s)+\beta \psi(s)] h(s) \\
& =-c_{1} \beta-c_{2} \delta-\sum_{-\infty}^{s=t} u(s) h(s)
\end{aligned}
$$


Likewise

$$
W_{t}(y, v)=c_{1} \alpha+c_{2} \gamma-\sum_{-\infty}^{s=t} v(s) h(s)
$$

From (2.13) and (2.14) we get

$$
\begin{aligned}
& W_{-\infty}(y, u)=-c_{1} \beta-c_{2} \delta, \quad W_{-\infty}(y, v)=c_{1} \alpha+c_{2} \gamma \\
& W_{\infty}(y, u)=-c_{1} \beta-c_{2} \delta-\sum_{s=-\infty}^{\infty} u(s) h(s) \text { and } \\
& W_{\infty}(y, v)=c_{1} \alpha+c_{2} \gamma-\sum_{s=-\infty}^{\infty} v(s) h(s)
\end{aligned}
$$

Substituting the values $W_{-\infty}(y, u)$ and $W_{-\infty}(y, v)$ from (2.15) into the first condition of (2.2) we obtain $-c_{2}(\alpha \delta-\beta \gamma)=0$, that is, $-c_{2} \omega=0$. Therefore $c_{2}=0$ and (2.11), (2.16) become

$$
\begin{gathered}
y(t)=c_{1} \varphi(t)+\frac{1}{\omega} \sum_{-\infty}^{s=t-1}[\varphi(s) \psi(t)-\varphi(t) \psi(s)] h(s) \\
W_{\infty}(y, u)=-c_{1} \beta-\sum_{s=-\infty}^{\infty} u(s) h(s), \quad W_{\infty}(y, v)=c_{1} \alpha-\sum_{s=-\infty}^{\infty} v(s) h(s)
\end{gathered}
$$

Substituting the values $W_{\infty}(y, u)$ and $W_{\infty}(y, v)$ from $(2.18)$ into the second condition of (2.2) we get

$$
c_{1}(-\gamma \beta+\alpha \delta)-\sum_{s=-\infty}^{\infty}[\gamma u(s)+\delta v(s)] h(s)=0
$$

Hence $c_{1}=(1 / \omega) \sum_{s=-\infty}^{\infty} \psi(s) h(s)$. Putting this value of $c_{1}$ in (2.17) we obtain

$$
y(t)=\frac{1}{\omega} \sum_{s=t}^{\infty} \varphi(t) \psi(s) h(s)+\frac{1}{\omega} \sum_{-\infty}^{s=t-1} \varphi(s) \psi(t) h(s),
$$

that is, (2.9) and (2.7) hold. The theorem is proved.

COROLLARY 2.2. The unique solution $y(t)$ of the nonhomogeneous equation (2.1) under the nonhomogeneous boundary conditions

$$
\alpha W_{-\infty}(y, u)+\beta W_{-\infty}(y, v)=d_{1}, \quad \gamma W_{\infty}(y, u)+\delta W_{\infty}(y, v)=d_{2}
$$


is given by the formula $y(t)=r(t)+\sum_{s=-\infty}^{\infty} G(t, s) h(s)$, where the function $G(t, s)$ is defined by (2.7), and

$$
r(t)=\frac{d_{2}}{\omega} \varphi(t)-\frac{d_{1}}{\omega} \psi(t) .
$$

PROOF. The function $r(t)$ defined by $(2.20)$ is a unique solution of the homogeneous equation (1.4) satisfying, by (2.4) and (2.5), the nonhomogeneous boundary conditions (2.19), while the function $\sum_{s=-\infty}^{\infty} G(t, s) h(s)$ is, by Theorem 2.1 , a unique solution of the nonhomogeneous equation (2.1) satisfying the homogeneous boundary conditions (2.2). Hence the desired result holds.

By Corollary 2.2 the nonlinear BVP (1.1), (1.8) is equivalent to the nonlinear equation

$$
y(t)=r(t)+\sum_{s=-\infty}^{\infty} G(t, s) f(s, y(s)), \quad t \in \mathbb{Z},
$$

where the functions $r(t)$ and $G(t, s)$ are defined by (2.20) and (2.7), respectively.

So we have to investigate the equation (2.21) in $\ell^{2}$. By (1.5), (2.8) and $r \in \ell^{2}$, we can define the operator $A: \ell^{2} \rightarrow \ell^{2}$ by the formula

$$
A y(t)=r(t)+\sum_{s=-\infty}^{\infty} G(t, s) f(s, y(s)), \quad t \in \mathbb{Z},
$$

where $y \in \ell^{2}$. Then (2.21) can be written as $y=A y$. Therefore solving (2.21) in $\ell^{2}$ is equivalent to finding fixed points of the operator $A$.

\section{The Lipschitz case}

In this section we will use the following well-known Contraction Mapping theorem, also known as the Banach fixed point theorem: Let $\mathscr{B}$ be a Banach space and $S$ a nonempty closed subset of $\mathscr{B}$. Assume $A: S \rightarrow S$ is a contraction, that is, there is $a \lambda, 0<\lambda<1$, such that $\|A u-A v\| \leq \lambda\|u-v\|$ for all $u, v$ in $S$. Then $A$ has $a$ unique fixed point in $S$, that is, a unique point $u_{0} \in S$ such that $A u_{0}=u_{0}$.

THEOREM 3.1. Assume conditions (C1)-(C3) are satisfied. In addition, let the function $f(t, \xi)$ satisfy the following Lipschitz condition: there is a constant $K>0$ such that

$$
\sum_{t=-\infty}^{\infty} \mid f(t, y(t))-f\left(t,\left.z(t)\right|^{2} \leq K^{2} \sum_{t=-\infty}^{\infty}|y(t)-z(t)|^{2}\right.
$$


for all $y$ and $z$ in $\ell^{2}$. If

$$
K\left\{\sum_{t=-\infty}^{\infty} \sum_{t=-\infty}^{\infty}|G(t, s)|^{2}\right\}^{1 / 2}<1,
$$

then the BVP (1.1), (1.8) has a unique solution in $\ell^{2}$.

ProOF. It will be sufficient to show that under the conditions of the theorem, the operator $A: \ell^{2} \rightarrow \ell^{2}$ defined by (2.22) is a contraction mapping. For $y, z \in \ell^{2}$ we have

$$
\begin{aligned}
|A y(t)-A z(t)|^{2} & =\left|\sum_{s=-\infty}^{\infty} G(t, s)[f(s, y(s))-f(s, z(s))]\right|^{2} \\
& \leq \sum_{s=-\infty}^{\infty}|G(t, s)|^{2} \sum_{s=-\infty}^{\infty}|f(s, y(s))-f(s, z(s))|^{2} \\
& \leq K^{2} \sum_{s=-\infty}^{\infty}|y(s)-z(s)|^{2} \sum_{s=-\infty}^{\infty}|G(t, s)|^{2} \\
& =K^{2}\|y-z\|^{2} \sum_{s=-\infty}^{\infty}|G(t, s)|^{2}
\end{aligned}
$$

for $t$ in $\mathbb{Z}$. Hence $\|A y-A z\| \leq \lambda\|\dot{y}-z\|$, where

$$
\lambda=K\left\{\sum_{t=-\infty}^{\infty} \sum_{t=-\infty}^{\infty}|G(t, s)|^{2}\right\}^{1 / 2}<1
$$

by (3.2). So, $A$ is a contraction mapping and the theorem is proved.

REMARK. The condition (3.1) is satisfied if $\left|f\left(t, \xi_{1}\right)-f\left(t, \xi_{2}\right)\right| \leq K\left|\xi_{1}-\xi_{2}\right|$ for all $t$ in $\mathbb{Z}$ and all $\xi_{1}, \xi_{2}$ in $\mathbb{R}$.

In the next theorem, the function $f(t, \xi)$ satisfies a Lipschitz condition not on the whole of $\ell^{2}$ but on a subset.

THEOREM 3.2. Assume conditions $(\mathrm{C} 1)-(\mathrm{C} 3)$ are satisfied. In addition, let there exist a number $R>0$ such that

$$
\sum_{t=-\infty}^{\infty}|f(t, y(t))-f(t, z(t))|^{2} \leq K^{2} \sum_{t=-\infty}^{\infty}|y(t)-z(t)|^{2}
$$


for all $y$ and $z$ in $S=\left\{u \in \ell^{2}:\|u\| \leq R\right\}$, where $K>0$ is a constant which may depend on $R$. If

$$
\left\{\sum_{t=-\infty}^{\infty}|r(t)|^{2}\right\}^{1 / 2}+\left\{\sum_{t=-\infty}^{\infty} \sum_{s=-\infty}^{\infty}|G(t, s)|^{2}\right\}^{1 / 2}\left\{\sup _{y \in S} \sum_{s=-\infty}^{\infty}|f(s, y(s))|^{2}\right\}^{1 / 2} \leq R
$$

and

$$
K\left\{\sum_{t=-\infty}^{\infty} \sum_{t=-\infty}^{\infty}|G(t, s)|^{2}\right\}^{1 / 2}<1,
$$

then the BVP (1.1), (1.8) has a unique solution $y \in \ell^{2}$ with $\sum_{t=-\infty}^{\infty}|y(t)|^{2} \leq R^{2}$.

Proof. Obviously, $S$ is a closed subset of $\ell^{2}$. Let $A: \ell^{2} \rightarrow \ell^{2}$ be the operator defined by (2.22). For $y$ and $z$ in $S$, taking into account (3.3) and (3.5), in exactly the same way as in the proof of Theorem 3.1, we can get $\|A y-A z\| \leq \lambda\|y-z\|$, where $\lambda<1$. It remains to show that $A$ maps $S$ into itself. For $y$ in $S$, we have

$$
\begin{aligned}
\|A y\| & =\left\|r(t)+\sum_{s=-\infty}^{\infty} G(t, s) f(s, y(s))\right\| \\
& \leq\|r\|+\left\|\sum_{s=-\infty}^{\infty} G(t, s) f(s, y(s))\right\| \\
& \leq\|r\|+\left\{\sum_{t=-\infty}^{\infty} \sum_{s=-\infty}^{\infty}|G(t, s)|^{2}\right\}^{1 / 2}\left\{\sum_{s=-\infty}^{\infty}|f(s, y(s))|^{2}\right\}^{1 / 2} \leq R
\end{aligned}
$$

by (3.4). Therefore $A: S \rightarrow S$.

Now the contraction mapping theorem can be applied to obtain a unique solution of (2.21) in $S$, and the proof is complete.

\section{Existence of solutions}

An operator (nonlinear, in general) acting in a Banach space is said to be completely continuous if it is continuous and maps bounded sets into relatively compact sets.

To get an existence theorem without uniqueness of solution, in this section we will apply the following Schauder fixed point theorem: Let $\mathscr{B}$ be a Banach space and $S$ a nonempty bounded, convex and closed subset of $\mathscr{B}$. Assume $A: \mathscr{B} \rightarrow \mathscr{B}$ is a completely continuous operator. If the operator $A$ leaves the set $S$ invariant, that is, if $A(S) \subset S$, then $A$ has at least one fixed point in $S$.

Passing on to the BVP (1.1), (1.8) let $A: \ell^{2} \rightarrow \ell^{2}$ be the operator defined by (2.22). 
LEMMA 4.1. Under the conditions (C1)-(C3) the operator $A$ is completely continuous.

PROOF. Consider $\epsilon>0$ and $y_{0} \in \ell^{2}$. We want to show that there exists $\delta>0$ such that

$$
y \in \ell^{2} \text { and }\left\|y-y_{0}\right\|<\delta \text { implies }\left\|A y-A y_{0}\right\|<\epsilon .
$$

We have $\left|A y(t)-A y_{0}(t)\right|^{2} \leq \sum_{s=-\infty}^{\infty}|G(t, s)|^{2} \sum_{s=-\infty}^{\infty}\left|f(s, y(s))-f\left(s, y_{0}(s)\right)\right|^{2}$. Hence

$$
\begin{aligned}
\left\|A y-A y_{0}\right\|^{2} \leq & M \sum_{s=-\infty}^{\infty}\left|f(s, y(s))-f\left(s, y_{0}(s)\right)\right|^{2} \\
= & M \sum_{|s| \leq N}\left|f(s, y(s))-f\left(s, y_{0}(s)\right)\right|^{2} \\
& +M \sum_{|s|>N}\left|f(s, y(s))-f\left(s, y_{0}(s)\right)\right|^{2},
\end{aligned}
$$

where $M=\sum_{t=-\infty}^{\infty} \sum_{s=-\infty}^{\infty}|G(t, s)|^{2}$ and $N$ is an arbitrary positive integer. Further, by the condition (1.5) and the elementary inequalities

$$
(a+b)^{2} \leq 2\left(a^{2}+b^{2}\right), \quad(a+b+c)^{2} \leq 3\left(a^{2}+b^{2}+c^{2}\right),
$$

we have

$$
\begin{aligned}
\sum_{|s|>N}\left|f(s, y(s))-f\left(s, y_{0}(s)\right)\right|^{2} \leq & \sum_{|s|>N}\left[|f(s, y(s))|+\left|f\left(s, y_{0}(s)\right)\right|\right]^{2} \\
\leq & \sum_{|s|>N}\left[2 g(s)+d|y(s)|+d\left|y_{0}(s)\right|\right]^{2} \\
\leq & \sum_{|s|>N}\left[12 g^{2}(s)+3 d^{2}|y(s)|^{2}+3 d^{2}\left|y_{0}(s)\right|^{2}\right] \\
\leq & 12 \sum_{|s|>N} g^{2}(s)+6 d^{2} \sum_{s=-\infty}^{\infty}\left|y(s)-y_{0}(s)\right|^{2} \\
& +9 d^{2} \sum_{|s|>N}\left|y_{0}(s)\right|^{2} .
\end{aligned}
$$

Choose $N$ such that $\sum_{|s|>N} g^{2}(s)<\epsilon^{2} /(48 M)$ and $\sum_{|s|>N}\left|y_{0}(s)\right|^{2}<\epsilon^{2} /\left(36 d^{2} M\right)$. Then we get

$$
\sum_{|s|>N}\left|f(s, y(s))-f\left(s, y_{0}(s)\right)\right|^{2}<\frac{\epsilon^{2}}{4 M}+6 d^{2} \delta^{2}+\frac{\epsilon^{2}}{4 M}
$$


After choosing $N$, by the continuity of $f(t, \xi)$ in $\xi$, we can find a $\delta_{0}>0$ such that $y \in \ell^{2}$ and $\left\|y-y_{0}\right\|<\delta_{0}$ imply

$$
\sum_{|s| \leq N}\left|f(s, y(s))-f\left(s, y_{0}(s)\right)\right|^{2}<\frac{\epsilon^{2}}{4 M} .
$$

Now setting $\delta^{2}=\min \left\{\epsilon^{2} /\left(24 d^{2} M\right), \delta_{0}^{2}\right\}$, we get from (4.2)-(4.4) the desired result (4.1). Thus, the operator $A$ is continuous. Next, let $Y \subset \ell^{2}$ be a bounded set: $\|y\| \leq c_{1}$ for all $y \in Y$. We must show that $A(Y)$ is a relatively compact set in $\ell^{2}$, that is, every infinite subset of $A(Y)$ has a limit point in $\ell^{2}$. To this end, we use the following known criterion for relative compactness in $\ell^{2}: A$ set $S \subset \ell^{2}$ is relatively compact if and only if $S$ is bounded and for every $\epsilon>0$ there exists a positive integer $t_{0}$ (depending only on $\epsilon)$ such that $\left.\sum_{|t|>t_{0}} \mid y(t)\right)\left.\right|^{2}<\epsilon^{2}$ for all $y \in S$. For all $y \in Y$, we have [see (3.6)]

$$
\|A y\| \leq\|r\|+\left\{M \sum_{s=-\infty}^{\infty}|f(s, y(s))|^{2}\right\}^{1 / 2} .
$$

On the other hand, using (1.5) we have

$$
\begin{aligned}
\sum_{s=-\infty}^{\infty}|f(s, y(s))|^{2} & \leq \sum_{s=-\infty}^{\infty}[g(s)+d|y(s)|]^{2} \leq 2 \sum_{s=-\infty}^{\infty}\left[g^{2}(s)+d^{2}|y(s)|^{2}\right] \\
& =2\left(\|g\|^{2}+d^{2}\|y\|^{2}\right) \leq 2\left(\|g\|^{2}+d^{2} c_{1}^{2}\right) .
\end{aligned}
$$

Therefore $\|A y\| \leq\|r\|+\left\{2 M\left(\|g\|^{2}+d^{2} c_{1}^{2}\right)\right\}^{1 / 2}$ for all $y \in Y$, that is, $A(Y)$ is a bounded set in $\ell^{2}$.

Further, for all $y \in Y$, we have

$$
\sum_{|t|>t_{0}}|A y(t)|^{2} \leq 2\left(\|g\|^{2}+d^{2} c_{1}^{2}\right) \sum_{|t|>t_{0}} \sum_{s=-\infty}^{\infty}|G(t, s)|^{2} .
$$

Hence we get by (2.8) that for a given $\epsilon>0$ there exists a positive integer $t_{0}$, depending only on $\epsilon$, such that $\sum_{|t|>t_{0}}|A y(t)|^{2}<\epsilon^{2}$ for all $y \in Y$. So, $A(Y)$ is a relatively compact set in $\ell^{2}$. The lemma is proved.

THEOREM 4.2. Assume conditions (C1)-(C3) are satisfied. In addition, let there exist a number $R>0$ such that

$$
\left\{\sum_{t=-\infty}^{\infty}|r(t)|^{2}\right\}^{1 / 2}+\left\{\sum_{t=-\infty}^{\infty} \sum_{s=-\infty}^{\infty}|G(t, s)|^{2}\right\}^{1 / 2}\left\{\sup _{y \in S} \sum_{s=-\infty}^{\infty}|f(s, y(s))|^{2}\right\}^{1 / 2} \leq R,
$$

where $S=\left\{y \in \ell^{2}:\|y\| \leq R\right\}$. Then the BVP (1.1), (1.8) has at least one solution $y \in \ell^{2}$ with $\sum_{t=-\infty}^{\infty}|y(t)|^{2} \leq R^{2}$. 
Proof. Let $A: \ell^{2} \rightarrow \ell^{2}$ be the operator defined by (2.22). By Lemma 4.1, the operator $A$ is completely continuous. Using (4.5), as in the proof of Theorem 3.2, we can see that $A$ maps the set $S$ into itself. Besides, it is obvious that the set $S$ is bounded, convex and closed. Therefore the Schauder fixed point theorem can be applied to obtain a solution of the equation (2.21) in $S$. The theorem is proved.

REMARK. Since for all $y$ in $S$ the left-hand side of (4.5) is less than or equal to

$$
\|r\|+\left\{\sum_{t=-\infty}^{\infty} \sum_{s=-\infty}^{\infty}|G(t, s)|^{2}\right\}^{1 / 2}\left\{2\left(\|g\|^{2}+d^{2} R^{2}\right)\right\}^{1 / 2},
$$

it follows that for a given $R>0$ the condition (4.5) will be satisfied if the numbers $d_{1}$ and $d_{2}$ in the boundary conditions (1.8), and the numbers $\|g\|$ and $d$ in the condition (1.5) are sufficiently small.

\section{References}

[1] R. P. Agarwal, M. Bohner and D. O'Regan, "Time scale systems on infinite intervals", Nonlinear Anal. 47 (2001) 837-848.

[2] R. P. Agarwal, M. Bohner and D. O'Regan, "Time scale boundary value problems on infinite intervals", J. Comput. Appl. Math. 141 (2002) 27-34.

[3] R. P. Agarwal and D. O' Regan, "Boundary value problems for general discrete systems on infinite intervals", Comput. Math. Appl. 33 (1997) 85-99.

[4] R. P. Agarwal and D. O'Regan, "Discrete systems on infinite intervals", Comput. Math. Appl. 35 (1998) 97-105.

[5] Yu. M. Berezanskii, Expansion in eigenfunctions of selfadjoint operators (Naukova Dumka, Kiev, 1965). English translation: (Amer. Math. Soc., Providence, RI, 1968).

[6] F. Dal and G. Sh. Guseinov, "Properties of discrete composition operators", J. Differ. Equations Appl. 11 (2005) 21-27.

[7] G. Sh. Guseinov, “A boundary value problem for second order nonlinear difference equations on the semi-infinite interval", J. Differ. Equations Appl. 8 (2002) 1019-1032.

[8] G. Sh. Guseinov and I. Yaslan, "Boundary value problems for second order nonlinear differential equations on infinite intervals", J. Math. Anal. Appl. 290 (2004) 620-638. 